\section{Geschichte der ärztlichen Verordnung von Opioiden an Abhängige}

Rainer Ullmann

Allgemeinarzt, Hamburg
Zusammenfassung: Es gab nie eine wissenschaftliche Begründung dafür, Opiaterhaltungstherapien zu verbieten. Opiaterhaltungstherapien (nicht Substitutionsbehandlungen) waren Standard bis in die 20er Jahre, weil die meisten Opiatabhängigen Abstinenz zu einem willkürlichen Zeitpunkt nicht erreichen konnten. Nach allgemeiner medizinischer Auffassung benötigen einige Patienten dauernd Opiate. Ein Teil der Morphinabhängigen der 20er Jahre war den Heroinabhängigen des letzten Drittels des 20. Jh. sehr ähnlich. Gerade bei dieser Gruppe hatten Entwöhnungsbehandlungen schlechte Ergebnisse, und Ärzte waren wenig interessiert, diese „Psychopathen“ zu behandeln. 1919 wurden in den USA und 1926 in Deutschland Opiaterhaltungstherapien verboten. Nach dem 2. Weltkrieg wurden die Ärzte vollständig aus der Behandlung Heroinabhängiger gedrängt, der ärztliche Gedanke der Schadensminderung galt nicht mehr. Behandlung war jetzt eine Mischung von Erziehung, Sozio- und Psychotherapie, bei der psychiatrische Begleitkrankheiten meist nicht angemessen behandelt wurden. Nach anfänglich euphorischer Betrachtung zeigten langfristige Katamnesen, daß die Behandlungsergebnisse so schlecht waren wie 50 Jahre zuvor. Wegen der HIV-Epidemie setzte sich das ärztliche Konzept der Schadensminderung seit Beginn der 90er Jahre langsam wieder durch. Jetzt sind Substitutionsbehandlungen (Ersatz des illegalisierten Heroins durch langwirkende Opioide in Dosierungen möglichst ohne pharmakologische Wirkung) die Standardbehandlung. Sie sind allerdings nach den Kriterien der sog. Abstinenzbehandlung und nach den staatlichen Kontrollbedürfnissen reglementiert.

Das Verbot der Erhaltungstherapien hat nicht zu einer Verringerung der Zahl der Opioidabhängigen geführt, sondern zu einer Zunahme auf etwa das 50fache. Die Sterblichkeit und das HIV-und Hepatitisinfektionsrisiko sind auf das 50-100fache - verglichen mit der übrigen Bevölkerung erhöht.

Schlüsselwörter: Opiatabhängigkeit - latrogener Morphinismus - Rolleston Komitee - Morphinverschreibungsverbot - Abstinenztherapie - Psychiatrische Komorbidität
History of Medical Maintenance of Opiate Dependents: There was never a scientific reason to prohibit opiate maintenance treatment. Opiate maintenance treatment (not the substitution of morphine or heroin through another opioid) was the standard treatment until the twenties. It was a general medical opinion that most of the opiate addicts could not remain abstinent at any arbitrary time and that some addicts would always need opiates. There was hardly any difference in the behaviour and appearance of a subgroup of morphine addicts from the twenties and the heroin addicts of today. Especially in this subgroup detoxification and residential treatment showed poor results. Medical doctors were not interested in treating those „psychopaths“. In 1919 the Supreme Court in the USA and in 1926 the „Reichsgericht“ prohibited opiate maintenance treatment. After World War II the physicians were entirely pushed out of the treatment of heroin addicts. Treatment was now a mixture of education, socio- and psychotherapy. Harm reduction was not allowed any more. There was no appropriate treatment for psychiatric co-morbidity. At the beginning they were very euphoric about the results of this treatment, but long term follow-up showed no better results than 50 years prior. The HIV-epidemic led to a renaissance of the medical concept of harm reduction during the last 10 years. Now the standard treatment is the substitution of the illegal heroin through long-acting opioids given in a dose not showing any pharmacological effect. However this treatment is not regulated according to medical science but to the goverments wish to control opiates. Prohibition of maintenance treatment led to an increase in the number of addicts, the risk of death, HIV- and hepatitisinfection of addicts up to 50 times.

Key words: Opiate Addiction - latrogenic Morphinism - Prohibition of Maintenance Treatment - Residential Treatment - Comorbidity - Rolleston Committee - Methadone Maintenance Treatment

Opiate waren in Europa jahrhundertelang weit verbreitet, gehörten in jede Hausapotheke und waren auch ein Genussmittel. Opiumsucht war trotzdem kein medizinisch diskutiertes Thema. Sucht wurde am Beispiel des Alkoholismus diskutiert. Opiumkonsumenten waren oft abhängig, aber meist sonst völlig unauffällig [1]. Opiumhaltige Arzneien wurden in den Apotheken und Drogerien viel verkauft. Die aufstrebende pharmazeutische Industrie bot zahllose opium-, morphinund kokainhaltige „Patentmedizinen“ an. Erst zu Beginn des 20. Jh. wurde sie gezwungen, die Inhaltsstoffe anzugeben,
Suchttherapie Sonderheft 2001; 2: 20-27

(c) Georg Thieme Verlag Stuttgart · New York ISSN 1439-9903 
etwa gleichzeitig wurden Apotheken- und dann Rezeptpflicht eingeführt.

Nach Einführung der Pravaz'schen Spritze 1864 entstand ein neues Krankheitsbild, die „Morphiumsucht“ [2]. Ursache war der unkritische Einsatz dieser neuen Behandlungsmethode durch die Ärzte. „Gäbe es keine Ärzte, gäbe es keinen Morphinismus“, urteilte Kraepelin. Besonders die morphinistischen Ärzte wurden zu „wahren Infektionsherden“ [3]. Zu dieser Zeit war der Gipfel des iatrogenen Morphinismus allerdings schon überschritten [4-6, für die USA 7].

Die Behandlung der Morphiumsucht war schwierig. Die Vorstellung: „Spritze weg, Morphium weg“ [6] erwies sich als falsch. Die Ärzte bemühten sich sehr um die Kranken, überwiegend Kollegen und ihre Frauen und bessere Stände, und sie versuchten, ihnen den Entzug so angenehm wie möglich zu machen. Sie litten mit ihren Patienten beim Entzug: Krafft-Ebing [8] schreibt 1897: „Die plötzliche (Entziehung) ist grausam, nicht ungefährlich und lässt Recidive noch mehr besorgen."

In diesen Jahren wurde die Behandlung Morphin- und Kokainsüchtiger lebhaft diskutiert. Die Verordnung von Kokain an Kokainabhängige wurde einhellig abgelehnt (plötzliches Absetzen sei ohne Gefahr für den Abhängigen möglich), aber über die Rezidivrate gab es keine Aussagen. Bei der Verschreibung von Morphin gingen die Meinungen auseinander. Die Mehrzahl bevorzugte den plötzlichen Entzug, eine starke Minderheit den Entzug mit absteigenden Morphindosierungen über 7-14 Tage, fast alle hielten eine geschlossene Abteilung für notwendig. Verordnungen von Morphin bis zum Entzug in möglichst niedriger Dosierung waren üblich und soziale Gründe für den Aufschub eines Entzuges wurden akzeptiert. Joel und Fraenkel weisen darauf hin, „dass die brüske Entziehung zuweilen der Ausdruck jener vorhin gekennzeichneten brüsken Stimmung des Arztes ist, in der er sich nicht so sehr als Helfer, wie als Richter fühlt“ [9]. Speer schreibt dazu: „Ich habe Psychiater als Grund für ihr Verhalten der plötzlichen Entziehung ohne jegliche Einschiebung eines anderen Pharmakons oft genug anführen hören, dass der vom Patienten dabei erlittene ungeheure Schock außerordentlich erzieherisch wirke, nämlich im Sinne der Abschreckung. Es ergibt sich also die eigentümliche Feststellung, dass Psychiater - also Seelenärzte - zu einer Zeit noch an die erzieherische Wirkung abschreckender Methoden glaubten, in der die Pädagogen sie so gut wie verworfen haben und in der man auch im Strafvollzug immer mehr von abschreckenden Methoden zu positiver Erziehung übergehen will“ [10].

Dem unangenehmen, durch ärztliche Hilfe gelegentlich erleichterten Entzug folgte der Rückfall fast $100 \%$ ig. Auch mehrmonatige psychotherapeutische Entwöhnungsbehandlungen führten nur bei einigen Prozent zur Abstinenz [3]. Die prognostischen Aussagen waren „zweifelhaft“ bis "höchst ungünstig“ [2,11-14]. „Bei einem Teil der Kranken ist von vornherein die Aussichtslosigkeit einer Dauerheilung offensichtlich" [15]. Es blieb nichts übrig, als vielen das Opiat weiterzuverschreiben, in möglichst geringer Dosis, und gelegentlich erneut Entzüge zu versuchen. Das war die Standardbehandlung bei den iatrogenen Morphinisten um 1900 und auch bei der Morphinismuswelle nach dem 1. Weltkrieg. Die ersten Katamnesen, die 1927 veröffentlicht wurden, zeigten überraschende Ergebnisse. Nach bis zu 13 Jahren waren unerwartet viele - 35\% der 211 Patienten - mehr als $1 \mathrm{Jahr}$ abstinent. Bei jungen Männern waren es nur $22 \%$. Bei den so genannten klassischen Morphinisten - Menschen, die erst im Erwachsenenalter und vollständig sozialisiert, häufig mit überdurchschnittlicher Produktivität, die wegen Krankheit oder wegen der leistungssteigernden Wirkung des Morphins zu Morphinisten geworden waren - waren die Ergebnisse besser. Auch spätere Katamnesen zeigten, dass die Prognose bei verschiedenen Gruppen unterschiedlich war, besonders schlecht bei jungen Menschen ohne vorherige soziale Integration. 25\% waren in dieser Zeit gestorben, $40 \%$ der Männer $[16,17]$. Regelmäßiger Morphinkonsum behinderte aber das soziale Leben und berufliche Höchstleistungen nicht zwangsläufig. $[2,18,19,14]$. Louis Lewin [20] schätzte bereits 1891 die Situation kritischer ein, und als die Morphinisten nicht mehr überwiegend Ärzte und Angehörige der Heilberufe waren, wurden sie von ärztlicher Seite nicht mehr so wohlwollend beurteilt.

Körperliche Schäden der Opiatabhängigkeit konnten in einer großen Untersuchung in den USA nicht nachgewiesen werden: „Außer der Abhängigkeit haben wir keine deutliche Abweichung der körperlichen Leistungsfähigkeit entdeckt" [21]. Dasselbe beschreiben Dole und Nyswander 40 Jahre später für Methadon [22]. Weil Morphinisten völlig unauffällig sein konnten, hatte Kraepelin schon 1910 eine mehrtägige Quarantäne vorgeschlagen, um den Erfolg der Entziehungskuren nach einiger Zeit zu überprüfen [3].

Man erkannte, dass es einige Morphiumsüchtige gab, die vielleicht lebenslang Opiate einnehmen müssen, damit sie „normal“ sind [14,23]. Dieser Gedanke ist später von Khantzian als „Selbstbehandlung“ wieder aufgenommen worden [24]. Außerdem wurde deutlich, dass Morphin langfristig oft notwendig blieb, wenn einmal Morphinismus entstanden war [25].

Die Epidemie nach dem 1. Weltkrieg wurde durch reichlichen Morphiumgebrauch im Krieg, durch Abgabe aus der Apotheke ohne oder gegen offensichtlich gefälschte Rezepte gefördert [26], später durch einen zunehmenden Schwarzmarkt, u.a. durch die fehlende Kontrolle bei der Produktion und „die Gewinnsucht ... des chemischen Kapitals“ [23,27-29]. Die Ärzte weigerten sich zunehmend, an Abhängige zu verschreiben [30]. Nur einzelne Ärzte bereicherten sich offensichtlich an den Morphinisten [29].

Insgesamt war die Zahl der iatrogenen Morphinisten im Vergleich zur Gesamtzahl der mit Morphin Behandelten sehr gering [16], wie es auch heute bei der Schmerztherapie bekannt ist. Bei den Kriegsbeschädigten wurde das Verhältnis mit 1:1000 errechnet [31].

In den 20er Jahren wurden die ersten Erhebungen angestellt, wie viele Morphinisten es gab [32]. Das war nach der Einführung der Rezeptpflicht, aber vor dem Verbot der Erhaltungstherapien. Man fand $0,056 \%$ bis $0,1 \%$ Morphinisten in Deutschland, in Hamburg 0,126\%, in Berlin 0,191\%. (Zum Vergleich: Nach 75 Jahren Heroinprohibition und Verbot der Opiaterhaltungstherapien gibt es jetzt etwa 3\%。 Heroinabhängige in Deutschland [33], 6,6\% in Hamburg [34]. 
Während zuerst Menschen mit chronisch schmerzhaften Krankheiten Morphin injizierten und in einigen Fällen davon abhängig wurden $[35,36]$, kamen später die Neurastheniker dazu [6] und dann die Psychopathen [37]. Bereits vor dem 1. Weltkrieg wurden verwahrloste und aus den sozialen Bezügen gefallene Morphinisten mit einer Minderung der geistigen Leistungsfähigkeit, auch einer ethischen und ästhetischen Abstumpfung beschrieben $[3,38]$. Jetzt änderte sich der Ton der Ärzte gegenüber den Süchtigen. Bei Bumke [39] sind die meisten Morphinisten „von Haus aus nervös minderwertige und sozial wenig brauchbare Menschen ... die Kranken zeigen von Hause aus die leidenschaftliche Neigung zum Missbrauche der verschiedensten Genussmittel, des Alkohols, Tabaks, Kaffees, unter Umständen auch anderer, ihnen gelegentlich verordneter Arzneimittel.“

Joel und Fränkel schreiben deshalb 1925: „Immer wieder haben uns solche Kranken geklagt, dass sie durch den barschen, bitteren und oft cynischen Ton, in welchem man sie weniger befragte, als verhörte und durch die schlechte Prognose, die man ihnen schon am ersten Tage stellte („Wenn Sie herauskommen, spritzen Sie ja doch gleich wieder ...“) aufs schlimmste beschämt und entmutigt wurden. ... Der Arzt interessiert sich mehr für die „Schuld“ des Patienten als für die Ursache seines Leidens. ... Vorschläge zu einer umfassenden Vorbeugung des Giftmissbrauches begegnen zuweilen einer gewissen Skepsis, weil es sich bei den Süchtigen um Psychopathen handele, die nun einmal ihren absonderlichen Weg gehen müssen; die gesellschaftlich wertvolleren Typen seien jedoch nicht in dem Maße gefährdet. Diese Auffassung ist nur zum kleinsten Teile richtig und in ihren Konsequenzen sehr bedenklich. Ganz abgesehen, davon, dass heutzutage der Psychopathenbegriff derart erweitert ist, dass man schließlich bei jedem Menschen einige psychopathische Züge finden dürfte, wird vielfach, wenn sich nichts anderes nachweisen läßt, die Sucht selber als Degenerationsmerkmal betrachtet, womit man sich natürlich im Kreise bewegt $[16,40]$.

Diese Autoren differenzieren die Morphinkrankheit (Morphinabhängigkeit) und die Morphinomanie (Morphiumsucht) in noch immer aktueller Weise: „Manche Irrtümer in der Behandlung der Morphinisten entspringen der unscharfen Erfassung dessen, woran sie eigentlich leiden. Die meisten Morphinsüchtigen leiden an zweierlei: erstens an einem pathologischen Trieb nach künstlicher Euphorie, der in verschiedenster Weise begründet sein kann und sich unter besonderen äußeren und inneren Umständen dem Morphin zuwandte. Sie leiden zweitens an chronischer Morphinvergiftung. Das Besondere und Komplizierende dieser zweiten Tatsache ist, dass die chronische Morphinvergiftung mit der allmählichen Erhöhung der Toleranz und dem Eintritt der Abstinenzerscheinungen bei Morphinmangel zu einem Hunger nach Morphin führt, der mit dem primären Verlangen nach euphorischen Giften nicht das Mindeste zu tun hat ... Morphinkrank kann jeder werden; Morphinomane zu werden setzt eine bestimmte Konstellation voraus, die übrigens gar nicht so selten ist. Die therapeutische Aufgabe gegenüber einem Morphinkranken ist die Entgiftung, gegenüber einem Morphinomanen über die Entgiftung hinaus eine Beeinflussung der gesamten Persönlichkeit ... Ohne damit die Berechtigung der therapeutischen Bemühungen um Entziehung im Geringsten infrage zu stellen, muss man sich doch darüber klar sein, dass beim eigentlichen Morphinomanen mit dem
Entzug ein leerer Raum geschaffen worden ist, den durch etwas Positives und Dauerndes auszufüllen nur in der Minderzahl der Fälle glücken wird. Es ist daher vorgeschlagen worden, sich gelegentlich mit einer Behandlung, die nur Reduktion der Morphinzufuhr zum Ziel hat, zu begnügen. So sehr dies wie eine halbe Maßnahme erscheint, ist ihre Anwendung mitunter nicht zu umgehen, ja in manchen, ganz bestimmten Fällen ist sogar eine periodisch sich wiederholende Reduktionsbehandlung das Zweckmäßigste.“

Diesen Gedanken nahm Kapuste [41] wieder auf: „Bei der so genannten Opiatsucht spielt der Heilmittelaspekt der Opiate die entscheidende Rolle. Es wäre denkbar, dass die meisten der „Süchtigen“ eine schwere Krankheit haben, die - nicht so einfach biochemisch nachweisbar wie der Diabetes - für den Patienten aber deutlich spürbar ist und auch deutlich spürbar durch Opiate gelindert wird ... Es ist relativ leicht, den durch Toleranz entstandenen hohen Tagesbedarf auf das normale Maß der Dosierung für nicht Süchtige zu senken, und relativ schwer, diese normale Dosis (... 30-40 Tropfen (L-Polamidon) pro Tag) endgültig abzusetzen. Wenn es sich um wirklich Süchtige handelte - möchte man meinen, könnte man die Dosis überhaupt nicht senken, weil ein Süchtiger immer nach mehr verlangt ... Man kann also, sofern man 3-4 Dosen pro Tag verschreibt, hunderte von so genannten Süchtigen ohne nennenswerte Probleme und mit deren weitgehend verlässlicher Kooperation bis zu diesem Punkt entziehen. Dann aber treten Probleme auf, nicht so sehr disziplinarische Probleme wie solche von Krankheitswert:

A sehr viele Depressionen, oft schwere, manchmal schwerste mit suizidalen Aktionen

B Psychosen, Angstzustände, Anfälle von Aggressivität und C Durchfallserkrankungen“.

Die Diskussion zur Komorbidität wurde bereits in den 20er Jahren geführt. Schwarz beschreibt 2 Sondertypen, die auch in der aktuellen Literatur beschrieben werden, wenn auch unter anderen Namen. Danach sei beim epileptoiden Typ (starke Reizbarkeit mit Neigung zu aggressiven jähzornigen Entladungen und asozialen Handlungen) die Prognose verschlechtert, während sie beim Typ der depressiven Konstitution und der hypomanischen eher besser sei [16]. Man schätzte 10-15 vom Hundert psychopathiefreie Morphinisten [37]. Auch heute werden bei genauer psychiatrischer Untersuchung 65\% psychisch gestörte Patienten unter den methadonbehandelten Heroinabhängigen gefunden [42,43].

Man wusste also bis 1930,

- dass bei medizinisch korrekter Anwendung von Opiaten selten Sucht entsteht

- dass Opiatsucht nicht kurzfristig durch Entzug geheilt werden kann

- dass auch mehrmonatige Behandlung (Trennung von der Droge und psychotherapeutische Kräftigung) nur Erfolgsraten von bestenfalls $20-35 \%$ hatte

- dass es auch um 1900 nicht nur „klassische Morphinisten“ gab und selbst die meist nicht entzogen werden konnten

- dass viele Opiatabhängige eine gewisse Dosis benötigen und dass es ihnen darunter gut geht,

- dass die geistige Leistungsfähigkeit unter einer konstanten Dosis im Toleranzbereich nicht beeinträchtigt ist 
- dass die Sterblichkeit der Morphinisten, die auf den illegalen Markt angewiesen waren, um 2\% pro Jahr lag (genau wie jetzt)

- dass die Sterblichkeit der Kriegsmorphinisten - die vom Staat das notwendige Morphin erhielten - erheblich geringer war

- dass durch Spritzen Krankheitserreger übertragen werden konnten, damals Syphilis und Tuberkulose [11].

Das Verbot der Erhaltungstherapien ist nur aus der Situation in den USA zu begreifen. Dort trat 1914 der Harrison Act in Kraft, der Produktion und Handel von Narkotika regelte. Wer Narkotika produzierte oder damit handelte, musste sich registrieren lassen und eine geringe Steuer zahlen. Narkotika wurden rezeptpflichtig gemacht, Besitz ohne ärztliche Verschreibung war strafbar. Ärzte waren von den Beschränkungen des Gesetzes ausgenommen, wenn sie „im Rahmen ihrer beruflichen Tätigkeit“" Narkotika verschrieben [44] Die Opiatabhängigen mussten zum Arzt und sich ein Rezept holen. [45]. Damit war das Medizinsystem überfordert. Die Verschreibung von Morphin, Heroin und Kokain war zunächst erlaubt, aber die meisten Ärzte weigerten sich, Abhängige zu behandeln. Einige bemühten sich um die Abhängigen, einige erkannten die Chance, viel Geld zu verdienen (Script doctors). Im Rahmen der Prohibitionsdiskussion drängte die Regierung auf ein Verschreibungsverbot an Abhängige. Die Scriptdoctors boten den Anlass, Ärzte wegen der Verschreibung vor Gericht zu stellen und „mit einem beispiellosen Eingriff Art und Umfang der ärztlichen Behandlung einzuschränken“ [46]. Sie strengte zu diesem Zweck mehrere Verfahren gegen Ärzte an, die Opiate an Abhängige verschrieben. Eine Verurteilung erreichte sie 1919. Der Supreme Court entschied, die Verordnung großer Mengen Morphin an einen Abhängigen sei ein Verstoß gegen das Gesetz. In einer Auslegung des Begriffes Rezept hatte das Gericht entschieden: „to call such order for the use of morphine a physician's prescription would be so plain a perversion of meaning that no discussion of the subject is required."

Folge dieses Urteils war, dass sich alle Ärzte, die an Abhängige verschrieben, strafbar machten, auch wenn sie versuchten, die Sucht zu behandeln. Vor dieser Entscheidung waren Erhaltungstherapien übliche Praxis gewesen. In den folgenden Jahren wurden Ärzte und Abhängige mit Strafverfahren überzogen, 3000 Ärzte und unzählige Abhängige wurden inhaftiert - ein fraglicher zivilisatorischer Fortschritt. Jetzt waren die Chancen vertan, Opiatabhängige medizinisch zu behandeln, und die Suchtmedizin fiel in einen Dornröschenschlaf. Damit war die schlechteste aller Lösungen geschaffen: verfügbare Opiate auf den Straßen ohne jede Kontrollmöglichkeit des Staates, Bestrafung der Abhängigen und Verbot, sie zu behandeln. 1917 wurde in New York der Kauf von Spritzen ohne ärztliches Rezept verboten [47]. Das war die gesetzliche Grundlage für die epidemische Verbreitung von AIDS und Hepatitis unter den Drogenabhängigen.

In Deutschland hatte sich das Reichsgericht der Entscheidung des Supreme Court angeschlossen. Es hatte am 5.10.1926 einen Arzt wegen Verstoß gegen das Opiumgesetz verurteilt, der innerhalb von 5 Monaten 3000 Rezepte über mindestens $3000 \mathrm{~g}$ Kokain ausgestellt und an Kokainschnupfer abgegeben hatte. Es hatte die Auffassung des Landgerichts Dresden verworfen, dass die ärztliche Rezeptur und die Abgabe von
Kokain auf ärztliche Rezepte grundsätzlich statthaft sei. Es hatte dagegen argumentiert, dass mit dem im Haager Opiumabkommen von 1912 verwendeten Begriff „medizinischer Gebrauch“ und dem Begriff „Heilmittel“ des Opiumgesetzes nicht jede ärztliche Anweisung gemeint sei, sondern nur die „Anwendung in ordnungsgemäßen Grenzen zur Schmerzstillung sowie die allmählich abnehmende Verabreichung an Kokainsüchtige zur Vermeidung der bei plötzlicher Entziehung auftretenden üblen Erscheinungen, nicht aber die regelmäßige Fortgewährung an Kokainsüchtige, wodurch diesen Kranken nicht geholfen, sondern geschadet wird.“ [88]

Diese juristische Entscheidung war damals wie heute nach medizinischer Kenntnis in zwei Punkten sachlich falsch. Fälschlich stellte das Reichsgericht Verschreibungen an Kokain- und Morphinabhängige rechtlich gleich; es hielt fälschlich bei Kokainsüchtigen „eine allmählich abnehmende Verabreichung zur Vermeidung der bei plötzlicher Entziehung auftretenden üblen Erscheinungen“ für notwendig, verbot aber mit dieser Entscheidung Dauerverschreibungen an Morphinabhängige. Dagegen versuchten sich Ärzte - wenn auch vergeblich - zu wehren. Alle Autoren äußerten die Ansicht, dass es Morphiumsüchtige gibt, denen nur mit der Verordnung einer geringen oder mittleren Dosis Morphium [27] ein normales Leben erhalten werden kann.

Abweichend davon konnten sich in Großbritannien die Ärzte durchsetzen. Im Bericht des „Rolleston Komitees“ von 1926 heißt es: Rückfall, früher oder später, scheint die Regel zu sein und die Heilung die Ausnahme in einer Größenordnung von $15-20 \%$. Dann definiert das Komitee die Indikationen für eine Dauerverschreibung von Morphin: Bei schweren Erkrankungen soll das Morphin gegeben werden, auch wenn es zur Abhängigkeit kommt. Bei 2 Gruppen von Abhängigen darf darüber hinaus dauernd Morphin verschrieben werden:

Wenn ein vollständiger Entzug ernsthafte Symptome produziert, die in der privaten Praxis nicht zufrieden stellend behandelt werden können, und bei denen, die mit einer bestimmten Menge ein normales und nützliches Leben führen können, aber sonst nicht (Departmental Committee). Damit hatte jeder Arzt das Recht, Heroin, Morphin und Kokain an Abhängige nach seiner Einschätzung zu verschreiben. In Deutschland wurde dagegen gefordert, Morphin „regelmäßig nur bei mit Schmerzen verbundenem, zum Tode führenden Siechtum “ zu verschreiben $[29,37,48]$. Entsprechend wurden in Deutschland seelisch und körperlich schwer Kranken Opiate vorenthalten. Der 47. Deutsche Ärztetag in Danzig beriet 1928 über die Gefahren der Rauschgifte für das deutsche Volk und ihre Bekämpfung; die folgende Resolution wurde angenommen:

„Bei der Behandlung des Morphinsüchtigen in der Praxis ist es wesentliche Aufgabe des Arztes, auf möglichst sofortige Einleitung einer Entziehungskur zu dringen.

Bis zum Beginn der Entziehungskur soll der Arzt mit möglichst geringen Dosen von Morphin auszukommen suchen ... Ist die Einleitung einer Entziehungskur aus äußeren Gründen oder mangels Einsicht oder guten Willens des Süchtigen nicht möglich, so liegt es im Interesse des Süchtigen wie auch des Arztes selbst, wenn der Arzt sich an eine Kommission aus sachverständigen Aerzten wendet, deren Einrichtung den 
Aerzteorganisationen empfohlen wird.“ [49]. Damals war es also Konsens, dass bei einer kleinen Gruppe Morphinabhängiger Opiaterhaltungstherapien notwendig sind. (1984 wurde beim 87. Deutschen Ärztetag beschlossen: „Die Ärzteschaft wird aufgefordert, den Abhängigen zu einer Behandlung zu motivieren.“ Aber abweichend von 1928 wird gefordert: „,... und bei bestehenden oder vorgetäuschten Entzugssyndromen keine Ersatzmittel zu verschreiben.“) [50]. Erst 1998 änderte die BÄK ihre Einstellung [51].

Zu Beginn der Drogenproblematik nach dem 2. Weltkrieg kamen die ersten Heroinabhängigen in die psychiatrischen Kliniken, die mit dem Problem überfordert waren. Deshalb wurden zunächst Selbsthilfegruppen, meist initiiert von ehemaligen Abhängigen, gefördert. Sucht wurde als Erziehungsdefizit oder als gesellschaftliches Problem angesehen. Der medizinisch übliche Ansatz, bei einer nicht kurzfristig heilbaren Krankheit, die meist nach Jahren ausheilt, lebensbedrohliche Komplikationen zu verhindern, wurde nicht akzeptiert. Es galt jetzt das Gesetz des Alles oder Nichts: Ohne vorherige Abstinenz, die oft ohne jede medikamentöse Erleichterung erreicht werden musste, gab es keine Behandlung noch so schwerer Krankheiten, weder körperlicher noch seelischer.

Im Vergleich mit der Behandlung anderer Krankheiten wird:

- der Zugang künstlich erschwert,

- bei Rezidiven der Behandlungsabbruch gefordert statt die Behandlung zu intensivieren (auch wenn die Behandlung schlecht, z.B. unterdosiert oder ohne notwendige Begleitmaßnahmen, durchgeführt wird),

- die medikamentöse Behandlung zusätzlicher psychischer Krankheiten erschwert oder gar verweigert.

Es werden zwingend Zusatzmaßnahmen gefordert (psychosoziale Betreuung), deren Wirksamkeit nicht für alle Heroinabhängigen notwendig [22,52,53] und erwiesen ist [54], von deren Inanspruchnahme aber die weitere Behandlung abhängig gemacht wird (oder es wird gar die Zulässigkeit der Substitutionsbehandlung bestritten, wenn die Kapazitäten der Zusatzbehandlung nicht ausreichen (10.BtMÄndV)). Vergessen wird dabei, dass auch die behandelnden Ärzte psychosoziale Betreuung leisten [55].

Obwohl die stationäre Abstinenztherapie bis heute den Beweis schuldig geblieben ist, dass sie effektiv ist (es gibt keine Untersuchung in Deutschland, die stationäre Abstinenztherapie gegen keine Behandlung oder gegen Substitutionsbehandlung vergleicht), durfte die Substitutionsbehandlung in den ersten Jahren nach ihrer massiv bekämpften Einführung [56-59] erst nach erfolglosen Abstinenztherapieversuchen begonnen werden.

Gorm Grimm [60] beschreibt das Suchtverständnis und die Praxis in sog. Abstinenztherapieeinrichtungen der 80er Jahre so: „Den Patienten werden in den betreffenden TherapieEinrichtungen nahezu alle bürgerlichen Rechte mit unglaublicher Selbstverständlichkeit entzogen; ein Gefängnisinsassse genießt weit mehr Rechte und Freiheiten. Der Patient ... wird geradezu als Kleinkind angesehen, das zu einem anderen Erwachsenen geformt werden soll ... In der ersten Phase, in der die völlige Isolierung von der Außenwelt und eine drastische Einschränkung der Entscheidungsfreiheit im In- nern der Einrichtung eine totale Kontrolle der Gemeinschaft über den Einzelnen verbürgt, wird die Regression des Klienten in den Status der Unmündigkeit und Abhängigkeit von anderen bewusst angestrebt.“

Die Behandlungserfolge dieser sehr eingreifenden Therapieform wurden zunächst nicht untersucht (DHS), und als sie untersucht wurden, waren sie nicht überzeugend: Über $60 \%$ hatten die Therapie abgebrochen [61]. 10-20\% derjenigen, die eine Therapie begonnen haben, blieben zeitweise oder dauernd abstinent [62]. Insgesamt waren das ca. $1 \%$ der Heroinabhängigen. Auch in Schweden wurden Ärzte aus der Behandlung gedrängt. Folge war die Verbreitung der HIV-Epidemie unter den Drogenabhängigen [63,64]. Die Vorstellung, dass es auch eine körperliche Ursache oder körperliche Folgen des chronischen Heroinkonsums geben könnte, die nicht innerhalb von Tagen rückgängig gemacht werden können und deshalb für viele Rückfälle verantwortlich sind, wurde nicht diskutiert.

Zur Heilung der Opiatabhängigkeit hatten Terry und Pellens 1928 [44] geschrieben: „Die Basis der vielfältigen Behandlungsmethoden ist die Trennung des Abhängigen von seiner Droge. Zur Heilung gehört, dass der Patient körperliche und seelische Integrität wiedererlangt. Dass dieses Ziel erreicht wird, ist der Literatur nicht zu entnehmen." Und ähnlich formulierte der Suchtforscher John Ball [65] fast 50 Jahre später: „Given the fact that no drug treatment program anywhere in the world has been able to secure voluntarily abstinence on the part of most opiate addicts, what can we say of present efforts and future prospects?“

Wegen der schlechten Ergebnisse der seit 1935 durchgeführten Entwöhnungsbehandlungen wurde in den USA in den 60ern eine medizinisch-medikamentöse Behandlung entwickelt, die die Restriktionen der Rechtsprechung berücksichtigte [22]: die regelmäßige fremdkontrollierte Einnahme eines langwirkenden Opioids, als Methadonsubstitution bekannt geworden. Niedergelassene Ärzte durften aber in den USA auch diese Behandlungsmethode nicht durchführen. Sie war nur außerhalb des medizinischen Systems in eigens dafür eingerichteten Methadonambulanzen erlaubt, deren Kapazitäten knapp gehalten wurden. Diese Behandlung wurde international intensiv untersucht. Überall - in den USA, in den Niederlanden, in Hongkong, Schweden, Australien und in vielen anderen Ländern, auch in Deutschland - hat sie viele positive Effekte: Sterblichkeit, HIV- und Hepatitisinfektionen und Kriminalität werden verringert, die soziale Rehabilitation wird ermöglicht [53].

In Deutschland wurde diese Behandlungsmethode sehr kritisch betrachtet. Noch mehr als 25 Jahre nach der Einführung wurden die guten Behandlungsergebnisse bestritten. Man setzte auf den Leidensdruck, was vielen Heroinabhängigen das Leben gekostet hat $[66,57]$. Die Behandlung mit Methadon wurde als chemische Zwangsjacke [67] oder „schlimmer als Freiheitsentziehung“" [68] diffamiert

Überhaupt nicht zu verstehen ist der Umgang der Ärzte mit Heroinabhängigen im Zusammenhang mit der AIDS-Epidemie. Bei jeder anderen Krankheit wäre alles getan worden, um eine lebensgefährliche und ansteckende Krankheit zu verhüten. Seit Beginn der AIDS-Epidemie sind die „Fixer“ [69] 
die zweitgrößte Gruppe der Infizierten. Einige der in dieser Zeit diskutierten Möglichkeiten zur Schadensminderung (die - medizinisch einleuchtend - die Zahl der Injektionen und damit das Infektionsrisiko mindern wie Spritzentausch, Substitutionsbehandlung, Konsumräume) wurden weitgehend abgelehnt oder nur mit erheblichen Einschränkungen genehmigt. Die Verhütung einer HIV-Infektion wurde als nicht so wichtig erachtet wie die Abstinenz, die vor Beginn der Therapie bestehen sollte. Bis 1990 gab es mehrere Publikationen, die die HIV-präventive Wirkung der Methadonsubstitution zeigten [70-75]. Aber die Ergebnisse dieser Publikationen wurden in Deutschland angezweifelt [76-78] bzw. ignoriert [56] und in den 1991 eingeführten NUB-Richtlinien war die Verhinderung der HIV-Infektion keine Behandlungsindikation. Ärzte, die Opioide verordneten, wurden disziplinarrechtlich und strafrechtlich verfolgt. Der einflussreiche Arbeitskreis „Ersatzdrogen“ der BÄK behinderte die Einführung massiv und ließ nur vereinzelte Indikationen wie „schwere konsumierende Krankheiten, am Ende der Schwangerschaft und unter der Geburt, AIDS-Kranke mit fortgeschrittener manifester Erkrankung“ gelten $[58,79]$. Nur bei diesen Indikationen musste die GKV die Kosten für die Behandlung übernehmen [80].

Wenn Heroinabhängige zur Linderung der Entzugssymptome andere Opioide von Ärzten wünschten, wurde das als Missbrauch diffamiert $[58,81]$. Ärztliche medikamentöse Behandlungen der Heroinabhängigkeit erschienen in der öffentlichen Diskussion nur als Verschreiben von „Ersatzdrogen“. Im Jahrbuch Sucht 1994 [82] wird diesem Problem ein Absatz im Kapitel „Rauschgiftsicherstellungen“ hinter dem Absatz „Illegale Labore“ gewidmet. Es dauerte noch einige Jahre, bis Substitutionsbehandlungen wenigstens unter „Schadensminderung“ eingestuft wurden.

Über viele Jahre lehnten die ärztlichen Funktionäre eine ärztliche und medikamentöse Behandlung der Heroinabhängigkeit ab. Ärzte hatten nur die Aufgabe, in spezielle therapeutische Einrichtungen und Programme zu vermitteln. Dass Heroinabhängigkeit eine auch durch Ärzte zu behandelnde Krankheit ist, war offensichtlich gar nicht denkbar [83]. Noch 2001 fragt der Vorsitzende des Bundesausschusses an, was denn der Inhalt der ärztlichen Behandlung bei der Heroinabhängigkeit sei. Die Einschätzung spiegelt sich auch in der Gebührenordnung wider: 10\% der Ausgaben für die Substitutionsbehandlung sind für die ärztlichen Gespräche vorgesehen, $90 \%$ für den vom Staat geforderten Kontrollaufwand.

Die Kontrolle ärztlicher Opiatverschreibungen und das Verbot der Erhaltungstherapien bei Opiatabhängigen hat 4 Effekte sicher gehabt:

1. die Zahl der iatrogenen Morphinisten hat drastisch abgenommen

2. die Zahl der morphinabhängigen Ärzte hat drastisch abgenommen

3. die Versorgung schmerzkranker Patienten hat sich drastisch verschlechtert

4. viele Opiatabhängige sind verelendet und gestorben, denen mit dieser Behandlung hätte geholfen werden können.
Das Verbot hat einen Effekt sicher nicht gehabt: Die Zahl der Opiatabhängigen ist nicht gesunken, sie ist drastisch gestiegen - auf etwa das 50fache gegenüber der Zeit, als das Verbot eingeführt wurde.

\section{Literatur}

${ }^{1}$ Berridge V. Opium and the People. London: Free Association Books, 1999; 36

${ }^{2}$ Levinstein E. Die Morphiumsucht. Berlin: Verlag von August Hirschwald Berlin, 1887; 10

${ }^{3}$ Kraepelin E. Psychiatrie - Ein Lehrbuch für Studierende und Ärzte. II. Band, Klinische Psychiatrie I. Teil. Leipzig: Verlag von Johann Ambrosius Barth, 1910; 8. Aufl.

${ }^{4}$ Bumke O. Lehrbuch der Geisteskrankheiten. München: Verlag von J. F. Bergmann, 1942; 5. Aufl.: 336

${ }^{5}$ Jacob W. Zur Statistik des Morphinismus in der Vor- und Nachkriegszeit. Archive f. Psychiatrie 1925; 76: 212-232

${ }^{6}$ Jastrowitz M. Die Deutsche Klinik am Eingange des 20. Jahrhunderts. Vorlesung über Morphinismus. Berlin und Wien: Urban und Schwarzenberg, 1906; VI. Band, 14: 414

${ }^{7}$ Courtwright DT. Dark Paradise. Opiate Addiction in America before 1940: Harvard University, 1982

${ }^{8}$ Krafft-Ebing R. Lehrbuch der Psychiatrie auf klinischer Grundlage. Stuttgart: Ferdinand Enke, 1897: 539

${ }^{9}$ Joel E, Fraenkel F. Zur Verhütung und Behandlung der Giftsuchten. Klinische Wochenschrift 1925; 4: 1713-1718

${ }^{10}$ Speer E. Morphiumentziehung im Dämmerschlaf. DMW 1931: 355-359

${ }^{11}$ Erlenmeyer A. Die Morphiumsucht und ihre Behandlung. Berlin, Leipzig, Neuwied: Heuser's Verlag, 1887; 3. Aufl.: 35-37

${ }^{12}$ Lewin L. Nebenwirkungen der Arzneimittel. Berlin: August Hirschwald, 1893: 136

${ }^{13}$ Bertololy C. Der Morphinismus und seine Behandlung. Frankfurt: Verlag Kritik, 1913: 33

${ }^{14}$ Binswanger-Jena O. Die Haager Internationale Opium-Konvention und Artikel 69 der Bundesverfassung. Schweizerische Med Wschr 1924; 54: 517-22

${ }^{15}$ Bonhoeffer K. Zur Therapie des Morphinismus. Therapie der Gegenwart 1926: 67

${ }^{16}$ Schwarz H. Über die Prognose des Morphinismus. Monatsschrift für Psychiatrie und Neurologie 1927; 63: 180-238

${ }^{17}$ Schwarz H. Weitere Untersuchungen zur Prognose des Morphinismus. Monatsschrift für Psychiatrie und Neurologie 1930; 84: 257-280

${ }^{18}$ Wolff P. Morphinismus und verwandte Alkaloidsuchten. Neue Deutsche Klinik. Berlin und Wien: Urban und Schwarzenberg, 1931; 7. Band: 533

${ }^{19}$ Bleuler E. Lehrbuch der Psychiatrie. Berlin, Göttingen, Heidelberg: Springer-Verlag, 1949; 8. Aufl.: 242

${ }^{20}$ Lewin L. Narkotische Genussmittel und die Gesetzgebung. Berliner Klinische Wochenschrift 1891: 1197-1201

${ }^{21}$ Light AB, Torrance EG. Opium addiction XI. General summary. Archives of Int Med 1929; 44: 870-876

22 Dole VP, Nyswander M. A Medical Treatment for Diacetymorphine (Heroin) Addiction. JAMA 1965; 193: 646-650

${ }^{23}$ Hösslin R. Ueber die Behandlung des Morphinismus. MMW 1924; 66: 1567-1569

${ }^{24}$ Khantzian E. Treating Addictiona as a Human Process. Northvale, New Jersey, London: Jason Aronson Inc., 1999

${ }^{25}$ Joel E, Fraenkel F. Öffentliche Maßnahmen gegen den Mißbrauch von Betäubungsmitteln. Klinische Wochenschrift 1927; 22: 1053-1057

${ }^{26}$ Bonhoeffer K. Über Verbreitung und Bekämpfung des Morphinismus und Kokainismus. Allg Zeitschrift für Psychiatrie 1926; 83: 238-249 
${ }^{27}$ Gaupp. Berichterstatter beim 47. Deutschen Aerztetag in Danzig. Aerztliches Vereinsblatt 1928; 57: 67ff

${ }^{28}$ Kauffmann A. Der Kokainismus und Morphinismus in der Kriegs- und Nachkriegszeit vom gerichtsärztlichen Standpunkt. Allgem Zeitschrift für Psychiatrie 1925; 80: 391-415

${ }^{29}$ Ilberg G. Über Verbreitung und Bekämpfung des Morphinismus und Kokainismus. Allgemeine Zeitschrift für Psychiatrie 1926; 83: 240-249

${ }^{30}$ Fallada H. Der tödliche Rausch. Bericht über das Glück, ein Morphinist zu sein. Neue Illustrierte 19.11.1955

${ }^{31}$ Dansauer und Rieth. Arbeit und Gesundheit, 1931; 18

32 Pohlisch K. Psychiatrisch-Neurologische Wochenschrift, 1931; 40

33 Bühringer G et al. Schätzverfahren und Schätzungen 1997 zum Umfang der Drogenproblematik in Deutschland. Sucht 1997; 43, Sonderheft 2

${ }^{34}$ Raschke P, Degkwitz P. Heroinkonsumenten: Verbreitung und Inanspruchnahme von Hilfen. In: Krausz M, Raschke P (Hrsg). Drogen in der Metropole. Freiburg im Breisgau: Lambertus, 1999

${ }^{35}$ Burkart R. Zur Pathologie der chronischen Morphiumvergiftung. DMW 1883; 9: 33-36

${ }^{36}$ König H. Die Prognose des Morphinismus. Klinische Wochenschrift 1914; 51: 1061-1064

${ }^{37}$ Meyer E. Über Morphinismus, Kokainismus und den Mißbrauch anderer Narkotika. Medizinische Klinik 1924; 20: 403-407

${ }^{38}$ Bumke O. Die Diagnose der Geisteskrankheiten. Wiesbaden: J. F. Bergmann, 1919: 350

${ }^{39}$ Bumke O. Lehrbuch der Geisteskrankheiten., 1924; 2. Aufl: 655

${ }^{40}$ Hahn B. Zur Behandlung der chronischen Morphiumerkrankung. Medizinische Klinik 1926; 28: 1068-1071

${ }^{41}$ Kapuste J. Mein Recht als Arzt auf Pflichterfüllung bei Behandlung psychischer Krankheiten. Teil III: Menschen, die keinen Arzt mehr finden: Institut für Ausbildungsforschung, 1994

42 Bender S, Scherbaum N, Finkbeiner Th, Rösinger C, Schall U, Lodemann E, Gastpar M. Psychiatrische Komorbidität bei Opiatabhängigen in Substitutionsbehandlung. In: Mann K, Buchkremer G (Hrsg). Suchtforschung und Suchttherapie in Deutschland. Sucht Sonderheft 1995: 133-135

${ }^{43}$ Krausz M, Verthein U, Degkwitz P. Komorbidität - Psychische Störungen und Symptome bei Opiatabhängigen. In: Krausz M, Raschke P (Hrsg). Drogen in der Metropole. Freiburg im Breisgau: Lambertus, 1999

${ }^{44}$ Terry Ch, Pellens M. The Opium Problem. Reprint 1970 der Ausgabe von 1928; 627

${ }^{45}$ Saper A. The Making of Policy Through Myth, Fantasy and Accident: The Making of America's Narcotic Laws. Br J Addict 1974; 69: 183-193

${ }^{46}$ de Ridder M. Heroin: Vom Arzneimittel zur Droge. New York: Campus Verlag, 2000: 113

${ }^{47}$ Musto D. The American Disease. New York, Oxford: Oxford University Press, 1987; 210

${ }^{48}$ Kahn E. Zur Zunahme des Morphinismus. MMW 1920; 20: 871-872

${ }^{49}$ Verhandlungen des 47. Deutschen Aerztetages. Leitsätze. Aerztliches Vereinsblatt für Deutschland 1928; 57: 66-67

${ }^{50}$ Entschließung des Deutschen Ärztetages DÄ, 1984; 81: B1783

${ }^{51}$ Flenker I. Pressestelle der deutschen Ärzteschaft, Pressekonferenz: Neue Wege in der Drogentherapie Bonn, 3. März 1998

${ }^{52}$ Raschke P, Verthein U, Kalke J. Substitution in Hamburg Methadonbehandlung Opiatabhängiger von 1990 bis 1995. Bericht zur Begleitforschung, 1996: 163

${ }^{53}$ Ward J, Mattick R, Hall W. Key issues in Methadone Maintenance Treatment. Kensington NSW, Australia: New South Wales University Press, 1992
${ }^{54}$ Kraft M, Rothbard A, Hyadley T, McLellan A, Asch D. Are Supplementary Services Provided During Methadone Maintenance Really Cost-Effective? Am J Psychiatry 1997; 154: 1214-1219

${ }^{55}$ Zenker C, Lang P. Methadon-Substitution in Bremen. Abschlussbericht der sozialmedizinischen Begleitforschung BIPS. Bremen: im Auftrag des Senators für Gesundheit, Jugend und Soziales, 1995

${ }^{56}$ Bühringer G. Überlegungen zur HIV-Prävention bei i.v.-Drogenabhängigen für die Sitzung des Ausschusses „Prävention“ des Nationalen AIDS-Beirates am 5. September 1989 in Bonn,: IFT, 31.8.1989

57 Täschner KL. Praktische Psychiatrie. Stuttgart: Kohlhammer, 1989: 201

${ }^{58}$ Keup W. Buprenorphin (Temgesic ${ }^{\circledR}$ )-Mißbrauch und Abhängigkeit. Suchtgefahren 1983; 29: 193-194

59 Bundesärztekammer. Beschluß des Vorstandes vom 9. Februar 1990: Ersatzdrogen. DÄ, 1990: B575-577

${ }^{60}$ Grimm G. Die Lösung des Drogenproblems. Flintbek: Buchverlag Wolf Plesser, 1985: $21 \mathrm{ff}$

${ }^{61}$ Ladewig D. Katamnesen bei Opiatabhängigkeit. In: Kleiner D. Langzeitverläufe bei Suchtkrankheiten (Hrsg). Berlin, Heidelberg, New York, London, Paris, Tokyo: Springer, 1987

${ }^{62}$ Raschke $\mathrm{P}$ und Schliehe F. Therapie und Rehabilitation bei Drogenkonsumenten, Langzeitstudie am Beispiel des „Hammer Modells“. Ministerium für Arbeit, Gesundheit und Soziales NRW 1985

${ }^{63}$ Gunne L. The Case of the Swedish Methadone Maintenance Treatment Programme. Drug and alcohol dependence 1983; 11: 99-103

${ }^{64}$ Gunne L. Politicians and scientists in the combat against drug abuse. Drug and alcohol dependence 1990; 25: 241-244

${ }^{65}$ Ball J. On the Treatment of Drug Dependence. Amer J Psychiat 1972; 128: 873-874

${ }^{66}$ Rubner O. Ärztliche Praxis. 1973; 25: 1784-1787

${ }^{67}$ Franke W. Übergeordnete Gesichtspunkte zur Bewertung von Methadonprogrammen. Suchtgefahren 1985; 31: 95-100

${ }^{68}$ Wanke K. Drogenabhängigkeit und Alkoholismus 1984. Tagungsberichte; Band 7

${ }^{69}$ BGA Dokumentation. AIDS-Forschung (AIFO) 1986; 176, 280

${ }^{70}$ Abdul-Quader AS et al. Methadone Maintenance and behaviour by intravenous drug users that can transmit HIV. Contemporary Drug problems 1987; 14: 425-434

${ }^{71}$ Marmor $\mathrm{M}$ et al. Risk factors for infection Human immunodeficiency virus among intravenous drug users in New York City. AIDS 1987; 1: 39-44

${ }^{72}$ Hartel D et al. Methadone Maintenance Treatment and reduced risk of AIDS and AIDS-specific mortality in intravenous drug users. IVth Conference on AIDS Stockholm Abstract 8546, 1988

${ }^{73}$ DesJarlais DC et al. HIV-1 infection among intravenous drug users in Manhattan, New York City, from 1977 through 1987. JAMA 1989; 261: 1008-1012

${ }^{74}$ Bartwell A, Senay E, Marks R, White R. Patients successfully maintained With Methadone Escaped Human Immunodefieciency Virus Infection. Arch Gen Psychiatry 1989; 46: 957-958

75 Novick D et al. Absence of Antibody to Human Immunodeficiency Virus in Long-term, Socially Rehabilitated Methadone Maintenance Patients. Arch Intern Med 1990; 150: 97-99

${ }^{76}$ Böcker FM. Behandlung drogenabhängiger Patienten mit Methadon, Teil 2: AIDS-Prävention durch Drogensubstitution. Fortschr Med 1990; 109: 132-134

${ }^{77}$ Heckmann W. AIDS-Prävention in der Drogenszene. Bewährungshilfe 1989: 76-90

${ }^{78}$ Stark K, Messing K, Bienzle U. Drogenabhängigkeit und AIDS ist der Einsatz von Ersatzdrogen gerechtfertigt? AIDS-FORSCHUNG 1988: 81-88 
${ }^{79}$ Stellungnahme des gemeinsamen Arbeitskreises des wissenschaftlichen Beirates und des Ausschusses „Psychiatrie, Psychotherapie und Psychohygiene“ der Bundesärztekammer vom 4. Februar 1988. Deutsches Ärzteblatt 1990; 87: B576-577

${ }^{80} \mathrm{KBV}$. Substitution unter strengen Auflagen. Die Richtlinien zur Methadon-Substitution dienen den erkrankten Drogenabhängigen und dem Schutz der Kassenärzte. Dt Ärzteblatt 1991; 88: A 4011

${ }^{81}$ Goedecke H, Lander C, Menges K. Dihydrocodein/Codein - keine Mittel zur Substitution bei Drogenabhängigen. Bundesgesundheitsbl 1994; 37: 207-212

82 Jahrbuch Sucht 1994: 113 Ausweichmittel/Ersatzstoffe

${ }^{83}$ NUB-Richtlinien vom 2.7.1991

84 Departmental Committee on Morphine and Heroin Addiction Report. His Majestýs Stationery Office London, 1926

${ }^{85}$ DHS Drogenabhängigkeit (verantwortlich: Gerchow J, Hermle L, Kovar KA, Wanke K), 1995; 2. Auflage: 33

${ }^{86}$ Maddux JF, Desmond DP. Methadone Maintenance and Recovery from Opioid Dependence. American Journal Alcohol Drug Abuse 1992; 18: 63-74

${ }^{87}$ Reichsgericht I. Strafsenat 5.10.1926 (I 184/26)
Dr. med. Rainer Ullmann

Allgemeinarzt

Curschmannstraße 10

20251 Hamburg 